\title{
FATORES INTERFERENTES NA ALIMENTAÇÃO DE CRIANÇAS DE 17 A 25 MESES DE UMA CRECHE MUNICIPAL
}

\author{
Aspects interfering in the feeding of children \\ from 17 to 25 months of a municipal day-care center
}

\author{
Ana Paola Nicolielo ${ }^{(1)}$, Cecília Gross ${ }^{(2)}$, Giédre Berretin-Felix ${ }^{(3)}$, \\ Maria Aparecida Miranda de Paula Machado ${ }^{(4)}$
}

\begin{abstract}
RESUMO
Objetivo: verificar fatores interferentes no padrão de alimentação de crianças de 17 a 25 meses. Métodos: dados de avaliações fonoaudiológicas de todas as crianças do berçário $(n=14)$ de uma creche no município de Bauru/SP. Aplicação de questionário aos pais a respeito das condições de saúde geral e desenvolvimento neuromotor, aleitamento natural ou artificial, hábitos orais e problemas respiratórios. Analisou-se a morfofuncionalidade dos constituintes do sistema estomatognático e características clínico-fisiológicas da mastigação e deglutição. A análise estatística foi descritiva. Resultados: constatou-se alteração em diversos aspectos da saúde nas crianças (92,8\%); presença de má oclusão (28,6\%); alterações morfológicas para lábio $(21,4 \%)$ e língua $(21,4 \%)$; alterações de tonicidade de bochechas $(57,1 \%)$, mento $(21,4 \%)$ e lábios $(42,8 \%)$; respiração oral ou oronasal $(42,8 \%)$; alterações na mastigação $(35,7 \%)$ e na deglutição de sólido $(50 \%)$ e líquido $(42,8 \%)$. Conclusão: para esse grupo, a presença de hábitos orais, assim como as alterações morfológicas e de tonicidade influenciam o padrão de alimentação das crianças analisadas, evidenciando a necessidade de prevenir e detectar precocemente tais fatores para que a alimentação nas crianças ocorra de forma adequada, promovendo assim condições de saúde para um desenvolvimento hígido.
\end{abstract}

DESCRITORES: Comportamento Alimentar; Criança; Desenvolvimento Infantil; Promoção da Saúde

\section{INTRODUÇÃO}

No início da vida, é esperado que a criança receba exclusivamente o leite materno até, pelo menos, os seis meses de idade ${ }^{1}$. Além dos benefícios amplamente divulgados na literatura (nutricionais, imunológicos, emocionais e econômicosociais) ${ }^{2}$, o leite materno também promove efeitos

(1) Fonoaudióloga; Mestranda em Fonoaudiologia do Programa de Pós-Graduação da Faculdade de Odontologia de Bauru da Universidade de São Paulo, FOB-USP, Bauru, SP.

(2) Fonoaudióloga.

(3) Fonoaudióloga; Professora Doutora do Departamento de Fonoaudiologia da Faculdade de Odontologia de Bauru da Universidade de São Paulo, FOB-USP, Bauru, SP; Doutora em Fisiopatologia em Clínica Médica.

(4) Fonoaudióloga; Professora Doutora do Departamento de Fonoaudiologia da Faculdade de Odontologia de Bauru da Universidade de São Paulo, FOB-USP, Bauru, SP; Doutora em Saúde Pública.

Conflito de interesses: inexistente positivos na saúde, uma vez que está relacionado ao crescimento e desenvolvimento craniofacial e motor oral do bebê. A sucção desempenha importante papel, possibilitando desenvolvimento adequado das funções de respiração, mastigação, deglutição e fala, reduzindo a probabilidade de ocorrência de alterações fonoaudiológicas ${ }^{3-5}$. Outro benefício demonstrado na literatura referese à habilidade cognitiva e o desempenho escolar, uma vez que a duração crescente da amamentação natural foi associada ao aumento estatisticamente significante no quociente de inteligência, compreensão da leitura, da matemática e outras habilidades escolares ${ }^{6}$.

Em alguns casos o aleitamento materno exclusivo pode estar prejudicado como em recém nascido pré-termo (RNPT), em que ocorre, especificamente, uma imaturidade global, incluindo o sistema estomatognático, que dificulta a realização da função de sucção e, consequentemente, a alimentação por via oral ${ }^{7-11}$. 
O desmame precoce, ou seja, antes dos seis meses de vida, ou a sucção não-exercitada, pode contribuir para a instalação de hábitos orais deletérios ${ }^{12}$, cujos mais frequentes (mamadeira, chupeta, sucção digital e respiração oral), geralmente, apresentam relação com a presença de má oclusão, além de poderem acarretar alterações nas funções de deglutição, respiração e fala ${ }^{13}$.

Referente à funcionalidade da respiração, o modo nasal propicia adequado crescimento e desenvolvimento do complexo craniofacial interagindo com outras funções como mastigação e deglutição ${ }^{14}$. Qualquer obstáculo à passagem do ar, pelas vias aéreas superiores, resultará em obstrução nasal obrigando a respiração tornar-se oral, prejudicando o desenvolvimento dessa região ${ }^{15}$. Obstruções nasais e/ou orolaríngeas parciais, com quadros permanentes ou descontínuos, possibilitam a posição alterada de língua e manutenção de lábios entreabertos ${ }^{16}$. Estudo indica que crianças respiradoras orais apresentam um menor período de aleitamento materno e um histórico de hábitos orais quando comparadas às crianças respiradoras nasais ${ }^{17}$.

No que diz respeito à alimentação, alguns indivíduos preferem alimentos umidificados e de consistência macia que diminuem a participação da musculatura orofacial constituindo provável fator etiológico ou contribuinte à existência do apinhamento dentário ${ }^{18}$, alteração da tonicidade, boca aberta, língua entre as arcardas e ceceio anterior ${ }^{19}$. Nesse sentido, é importante estimular a criança pequena a mastigar alimentos mais consistentes, com a finalidade de preparar a musculatura orofacial para os movimentos precisos e coordenados, necessários para a deglutição madura e para a fala ${ }^{17}$.

Atualmente, o modernismo faculta a possibilidade dos genitores entregarem, durante o dia, os cuidados alimentares de seus filhos a uma determinada instituição, sem que saibam ou tenham participação na composição do cardápio cotidiano. Além disso, crianças matriculadas em creches estão expostas a fatores que alteram o desenvolvimento normal do sistema estomatognático e, consequentemente, de suas funções. Dessa forma, o presente estudo teve como objetivo caracterizar fatores interferentes no padrão de alimentação de crianças de 17 a 25 meses que frequentavam o berçário de uma creche no município de Bauru-SP.

\section{MÉTODOS}

Foram selecionados dados dos prontuários de todas as crianças matriculadas no berçário da creche em questão $(\mathrm{N}=14)$, referentes às avaliações fonoaudiológicas dessas crianças, sendo quatro do sexo feminino $(28,5 \%)$ e dez do sexo masculino (71,4\%), com média de idade de 21,35 meses, que frequentavam o berçário de uma creche do município de Bauru-SP. Os critérios de exclusão adotados foram relacionados à presença de malformação craniofacial, distúrbios neurológicos e casos sindrômicos, os quais não foram verificados no local de desenvolvimento do estudo. Os dados foram coletados durante o estágio curricular referente à disciplina Fonoaudiologia Preventiva II do Curso de Fonoaudiologia da Faculdade de Odontologia de Bauru da Universidade de São Paulo, FOB/USP, durante o primeiro semestre de 2005, sendo todos os procedimentos de triagem e avaliação rotineiros à dinâmica da prática.

Foi aplicado um questionário aos pais das crianças, a fim de obter informações sobre os parâmetros pré-selecionados de condições de saúde geral (tempo gestacional, intercorrências no nascimento, alterações durante o sono, doenças pregressas, internações, cirurgias, tratamentos realizados e saúde oral); desenvolvimento neuromotor (sequência básica: firmar a cabeça, sentar, engatinhar, andar e falar); histórico de amamentação (tempo de amamentação natural e uso de mamadeira); ocorrência de hábitos orais (bruxismo, onicofagia, sucção digital e de chupeta); e problemas respiratórios (baba noturna, ronco e doenças respiratórias tratadas ou não). As crianças foram avaliadas sob dois aspectos: morfofuncionalidade dos constituintes do sistema estomatognático e características clínicas e fisiológicas da mastigação e deglutição.

Os sujeitos da pesquisa foram examinados, no que se refere aos aspectos anatômicos (lábios, língua, palato duro, palato mole, número de dentes e oclusão), por meio de observação; tonicidade e tensão (bochechas, mento, lábios e língua) e por meio de palpação, além de mobilidade e motricidade (lábios, língua, bochechas e mandíbula). Para avaliação dos aspectos anatômicos, da mobilidade e da motricidade foi solicitado à criança que mandasse um beijo, estalasse e mostrasse a língua e que abrisse a boca.

O modo respiratório foi avaliado por meio da observação da postura de mandíbula, lábios e língua das crianças durante as atividades rotineiras da creche. Além disso, utilizou-se o espelho de Glatzel posicionado inferiormente às narinas para investigação do fluxo aéreo expiratório. Foi atribuído o padrão adequado ao modo nasal caracterizado pela permanência de lábios ocluídos e fluxo expiratório simétrico durante o processo de observação. Foi considerada respiração oral ou oronasal quando a criança mantinha lábios desocluídos, 
mandíbula e língua abaixadas por todo ou parte do período de observação, somado à alteração do fluxo expiratório.

Para verificar o padrão alimentar de cada criança, foram realizadas filmagens durante a refeição das mesmas, considerando-se alimentos de diferentes consistências, usualmente oferecidos na própria creche, e posteriormente analisados os padrões de mastigação e deglutição.

Quanto à mastigação foram analisados: a apreensão do alimento, sendo adequada quando realizada com os dentes anteriores e inadequada quando partiam o alimento com as mãos ou mordiam lateralmente; o escape anterior observado quando havia escoamento de alimento ou de saliva pela rima bucal; o tipo mastigatório adequado foi o bilateral e inadequado quando a trituração do alimento ocorreu com os dentes anteriores ou unilateralmente; o tempo de mastigação foi verificado de forma subjetiva pelo examinador, considerado adequado quando a criança realizava o número de ciclos mastigatórios necessários para trituração do alimento em tempo hábil, sendo inadequado quando o tempo foi aumentado ou reduzido, podendo trazer prejuízo para a eficiência mastigatória; e, por fim, a coordenação entre mastigação, deglutição e respiração, que foi adequada quando não houve engasgos, tosse ou alterações do ritmo respiratório.

Foi avaliada a deglutição de líquido fino (água ou suco) e de sólido (bolacha recheada), considerando-se: movimentos adequados de língua quando internos à cavidade oral com efetiva ejeção do bolo alimentar, enquanto movimentos póstero-anteriores além da rima bucal, foram adotados como padrão inadequado: tempo do alimento na boca analisado subjetivamente, levando em conta a impressão do tempo necessário para a organização e propulsão do alimento; presença ou ausência de nasorregurgitação, alteração respiratória, modificação da coloração facial e tosse durante a deglutição dos alimentos oferecidos.

Este estudo teve aprovação do Comitê de Ética em Pesquisa da Faculdade de Odontologia de Bauru (Processo no 101/2005).

Os resultados foram submetidos a uma análise Estatística Descritiva, com o uso de frequências absolutas e relativas.

\section{RESULTADOS}

Com relação à saúde geral os resultados encontrados estão apresentados na Figura 1, sendo que os questionários respondidos pelos pais revelaram que o padrão de sono demonstrou maior ocorrência de alterações referidas, verificado para oito crianças $(58,3 \%)$. Além disso, prematuridade e doenças pregressas foram citadas para três crianças $(21,4 \%)$, enquanto intercorrências ao nascimento (anóxia/hipóxia e necessidade de ficar em incubadora) e uso de medicamentos estiveram presentes para quatro $(28,5 \%)$.

No que se refere ao desenvolvimento neuromotor, nenhuma criança apresentou alterações até o momento da avaliação quanto aos itens: firmar cabeça, sentar, engatinhar e andar. O aparecimento

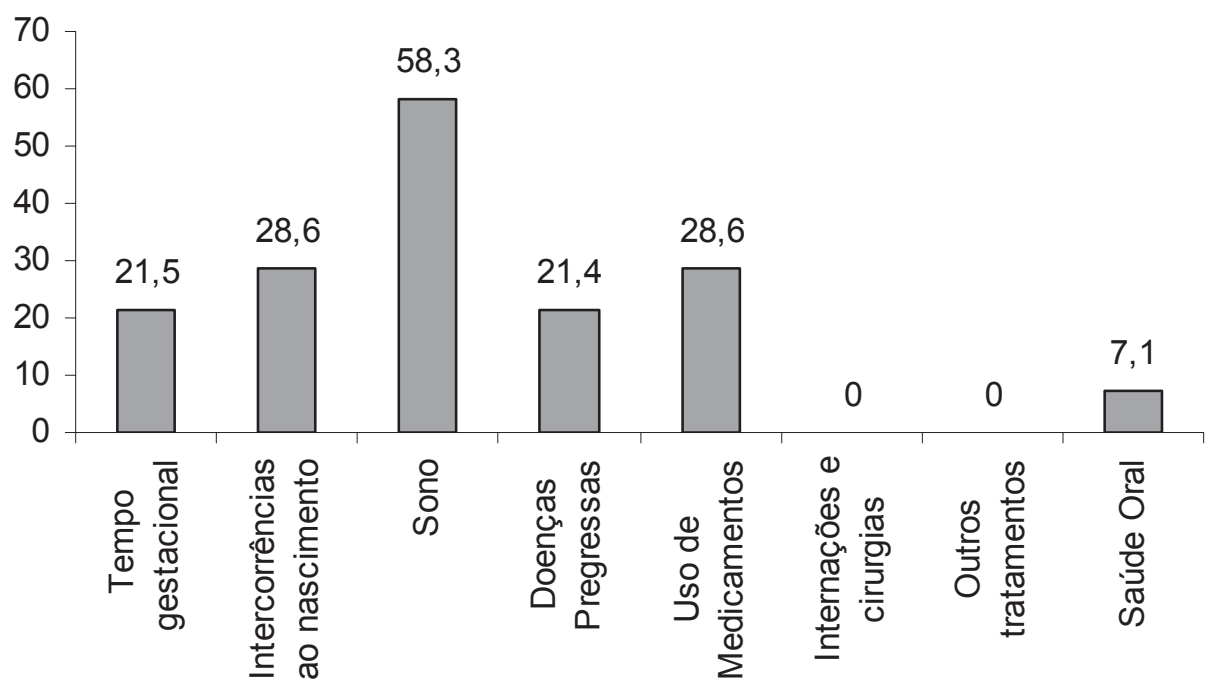

Figura 1 - Apresentação da porcentagem de ocorrência de queixas relacionadas à saúde geral das crianças estudadas 
das primeiras palavras apresentou-se inadequado para duas crianças $(14,3 \%)$.

Em relação ao histórico da amamentação, duas crianças receberam aleitamento materno por um período menor do que seis meses $(14,3 \%)$ e apenas uma criança não foi amamentada naturalmente.

No que diz respeito aos hábitos orais, segundo os pais, quatro $(28,5 \%)$ crianças utilizavam chupeta, a mesma porcentagem de crianças sugava 0 dedo e nenhuma apresentava onicofagia.

Quanto aos problemas respiratórios, verificou-se que, duas crianças $(16,6 \%)$ roncavam e uma $(8,3 \%)$ apresentava baba noturna. Segundo os pais, três $(21,4 \%)$ crianças apresentavam alergias respiratórias e quatro $(28,5 \%)$ utilizavam medicamentos como antipiréticos e broncodilatadores.

Os resultados da avaliação orofacial relacionados aos aspectos morfológicos revelaram que quatro $(28,6 \%)$ crianças apresentaram alteração dentoclusal, caracterizada por mordida aberta anterior, enquanto três $(21,4 \%)$ crianças tinham lábio inferior evertido, sendo que para esta mesma porcentagem foi encontrada alteração de língua, caracterizada por hipotonia (Figura 2).

No que se refere à tonicidade da musculatura orofacial, oito $(57,1 \%)$ apresentaram hipotonia de bochechas, três $(21,4 \%)$ apresentaram hipertonia do mento e seis (42,8\%) apresentaram hipotonia de lábios.

Quanto aos problemas respiratórios foi evidenciada respiração oral ou oronasal em seis $(42,8 \%)$ crianças.

Relativo à mastigação, dentre os aspectos avaliados, foi constatado escape anterior de alimentos para uma criança $(7,1 \%)$ e tempo mastigatório reduzido ou aumentado para quatro (28,6\%).

$\mathrm{Na}$ deglutição houve presença de movimento inadequado de língua para 5 crianças (35,7\%) quando analisado o alimento sólido e para 4 crianças $(28,6 \%)$ durante a alimentação com líquido. Já a presença de tosse ao deglutir foi verificada para duas crianças distintas (14,3\%), em ambas consistências testadas.

Os dados individuais da ocorrência de sinais e/ ou sintomas dos diferentes aspectos investigados, como também da presença de alterações nas funções de mastigação e deglutição das crianças estudadas encontram-se na Tabela 1. A análise dessa tabela demonstra que grande parte das crianças com problemas na mastigação e na deglutição de líquido apresentou hábitos orais, bem como alterações nos aspectos morfológicos e na tonicidade. Em relação à mastigação e deglutição de sólido também houve alterações concomitantes para as mesmas crianças.

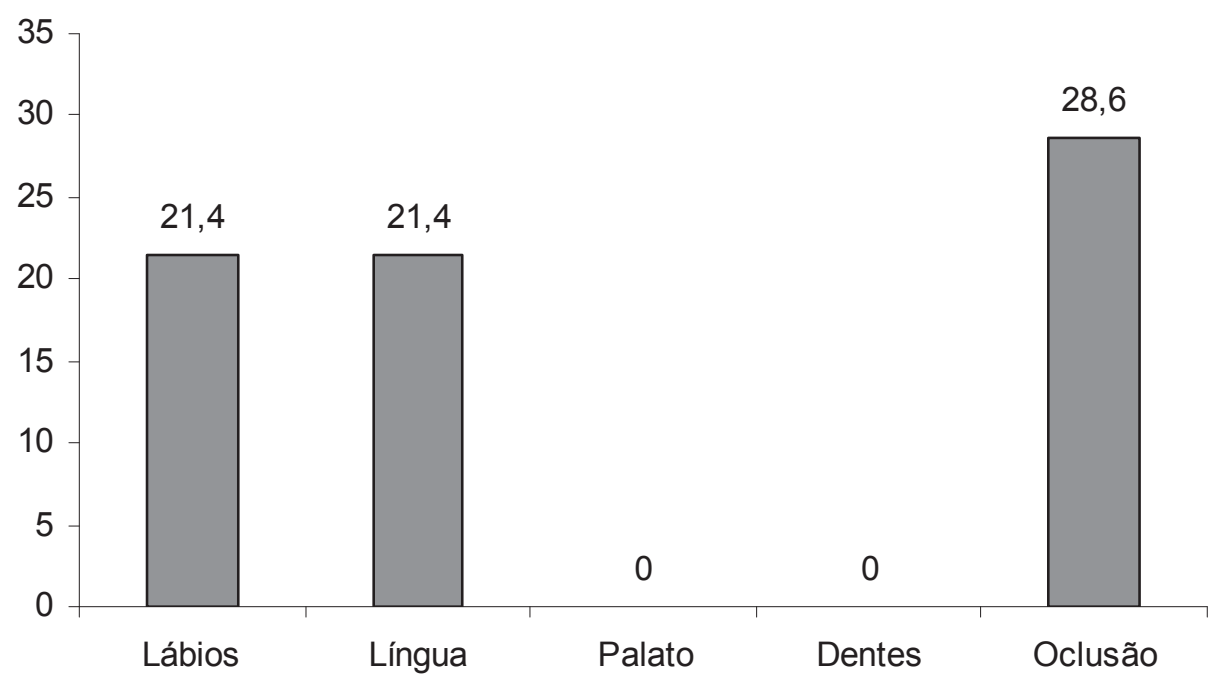

Figura 2-Apresentação da porcentagem de ocorrência de alteração quanto aos aspectos morfológicos do sistema estomatognático para as crianças estudadas 
Tabela 1 - Apresentação da ocorrência de alterações individuais obtidas por meio do questionário e da avaliação

\begin{tabular}{|c|c|c|c|c|c|c|c|c|c|c|}
\hline & \multicolumn{5}{|c|}{ Questionário } & \multicolumn{5}{|c|}{ Avaliação } \\
\hline & Saúde Geral & DNM & $\begin{array}{l}\text { Histórico } \\
\text { amamen- } \\
\text { tação }\end{array}$ & $\begin{array}{c}\text { Hábitos } \\
\text { Orais }\end{array}$ & $\begin{array}{c}\text { Problemas } \\
\text { respira- } \\
\text { tórios }\end{array}$ & $\begin{array}{l}\text { Aspectos } \\
\text { Morfoló- } \\
\text { gicos }\end{array}$ & Tonicidade & Mastigação & $\begin{array}{l}\text { Deglutição } \\
\text { sólido }\end{array}$ & $\begin{array}{l}\text { Deglutição } \\
\text { líquido }\end{array}$ \\
\hline 1 & $P$ & - & $P$ & $P$ & - & $P$ & $P$ & - & $P$ & - \\
\hline 2 & $P$ & - & - & - & - & $P$ & - & - & - & - \\
\hline 3 & $\mathrm{P}$ & - & - & $P$ & - & $P$ & $P$ & $P$ & $P$ & - \\
\hline 4 & $P$ & - & - & $P$ & $P$ & - & $P$ & $P$ & - & - \\
\hline 5 & $P$ & - & - & $P$ & - & - & $P$ & - & $P$ & - \\
\hline 6 & $P$ & - & - & $P$ & - & - & - & - & - & $P$ \\
\hline 7 & $P$ & - & - & $P$ & - & - & $P$ & - & - & $P$ \\
\hline 8 & $P$ & - & - & - & $\mathrm{P}$ & $P$ & $P$ & $P$ & $P$ & $\mathrm{P}$ \\
\hline 9 & - & - & - & - & - & $P$ & - & $P$ & $\mathrm{P}$ & - \\
\hline 10 & $P$ & - & - & $P$ & - & $P$ & - & - & - & - \\
\hline 11 & $P$ & - & - & $P$ & - & $P$ & $P$ & - & $P$ & $\mathrm{P}$ \\
\hline 12 & $P$ & $P$ & - & - & $P$ & - & - & - & - & $P$ \\
\hline 13 & $P$ & - & $P$ & - & - & $P$ & $P$ & $P$ & $\mathrm{P}$ & $\mathrm{P}$ \\
\hline 14 & $P$ & $P$ & $P$ & - & $P$ & $P$ & $P$ & - & - & - \\
\hline
\end{tabular}

Legenda: $\mathrm{DMN}=$ desenvolvimento neuromotor; $\mathrm{P}=$ presente; $-=$ ausente.

\section{DISCUSSÃO}

Após análise dos resultados optou-se por discorrer apenas a respeito dos aspectos que apresentaram alteração.

De acordo com a Organização Mundial de Saúde (OMS) ${ }^{1}, 10 \%$ dos nascimentos são de prematuros. No Brasil, estima-se que essa porcentagem possa ultrapassar $20 \%$, dependendo da região ${ }^{19}$. No estudo presente, esse indicador foi ultrapassado, visto que a ocorrência de nascimentos prematuros foi de $21,4 \%$. Vale ressaltar que a importância do peso ao nascimento, assim como da idade gestacional, é justificada pelo papel relevante que esses fatores desempenham na maturidade de vários sistemas em recém-nascidos.

Verificou-se que a maioria das crianças $(85,7 \%)$ apresentou alteração no sono. A classificação mais utilizada dos distúrbios do sono (em relação à vigília) é a da American Academy of Sleep Medicine 20 que aponta as alterações da respiração como um fator de tal distúrbio. A alteração de maior ocorrência encontrada diz respeito ao padrão de sono fragmentado $(58,3 \%)$, que, em parte, poderia ser justificado pelo fato de que, nesse estudo, foi relatado o uso do xarope com o princípio ativo Cetotifeno em $21,42 \%$ das crianças. Esse medicamento é indicado para a prevenção e tratamento de distúrbios alérgicos multissistêmicos e pode provocar alguns efeitos colaterais como irritabilidade, excitação, insônia e nervosismo ${ }^{21}$.

No presente estudo, apesar da diferença de faixa etária, foi constatado que $16,6 \%$ das crianças apresentaram ronco. Particularmente em crianças, estudos relatam que, entre as idades de 6 e 11 anos, a prevalência do ronco é de $11,2 \%{ }^{22}$. Há possibilidades do ronco e da baba noturna serem justificados pela respiração oral durante o sono devido às suas diversas causas, incluindo a obstrução das vias aéreas superiores ${ }^{23}$, visto que não há indícios de disfunções neurológicas.

Não há dúvidas de que a amamentação também desempenha um papel importante para o desenvolvimento da criança. Segundo a UNICEF ${ }^{20}$, nos últimos anos no Brasil, houve um crescimento significativo dos índices de aleitamento materno, sendo que os resultados deste trabalho constataram que a maioria das crianças $(78,57 \%)$ foi aleitada exclusivamente por um período igual ou maior do que o recomendado (180 dias). A literatura tem apontado a importância da sucção durante o aleitamento natural, pois promove o desenvolvimento adequado dos órgãos fonoarticulatórios quanto à mobilidade, força, postura, e o desenvolvimento das funções de respiração, mastigação, deglutição e articulação dos sons da fala ${ }^{4}$. Entretanto, vale ressaltar que, neste estudo, o aleitamento não teve comprovação de exclusividade e que, mesmo amamentadas por um período igual ou maior do que o recomendado constatou-se ocorrência de alterações morfológicas, de tonicidade e/ou nas funções de mastigação e/ou deglutição.

Para as crianças dessa pesquisa, os hábitos orais mais comuns foram à sucção digital ou de chupeta. Nesse sentido, um estudo ${ }^{24}$ identificou em crianças de seis anos de idade interação entre duração da amamentação e uso de chupeta com problemas de má oclusão. A literatura ${ }^{25,26}$ aborda, 
ainda, o hábito de sucção de chupeta como fator causal significante para o surgimento da mordida aberta anterior, o que foi encontrado para quatro crianças desse estudo, embora avaliadas em uma idade anterior à pesquisa citada.

Com relação à respiração, sabe-se que esta deve ser predominantemente nasal, quando em repouso ou silêncio. Quanto ao modo respiratório, os resultados da avaliação evidenciaram respiração oral ou oronasal em $42,8 \%$ das crianças. Apesar da diferença metodológica, a literatura demonstra que, muitas vezes encontram-se indivíduos que fazem uso do modo oral ou oronasal de respiração, seja por hábito vicioso ou por alterações orgânicas com obstáculos à passagem do ar pelas vias aéreas superiores ${ }^{23}$.

Constatou-se em $57,1 \%$ das crianças estudadas, hipotonia de bochechas e em 42,8\%, hipotonia de lábios e em $28,5 \%$, má oclusão dentária, caracterizada por mordida aberta anterior. Segundo a literatura, no respirador oral, a má oclusão dentária, a postura dos lábios entreabertos, a hipotonia labial e a postura alterada da língua são mudanças morfológicas que podem ocasionar distúrbios na mastigação e na deglutição ${ }^{27,28}$. De fato, neste estudo constataram-se alterações no tempo mastigatório, escape oral anterior de alimentos, movimento inadequado de língua e tosse na deglutição de sólidos e líquidos, que podem ser justificados pelas condições morfofuncionais alteradas. Além disso, os hábitos orais deletérios podem interferir negativamente no desenvolvimento das funções orofaciais ${ }^{23}$. Os resultados encontrados indicam a necessidade de prevenir e detectar precocemente a interferência de tais fatores na alimentação, de forma a promover condições de saúde para um desenvolvimento hígido das crianças.

\section{CONCLUSÃO}

Diante das informações referidas pode-se sugerir que hábitos orais, alterações morfológicas e de tonicidade podem influenciar negativamente $o$ padrão de alimentação das crianças analisadas, evidenciando a necessidade de prevenir e detectar precocemente tais fatores para que a alimentação nas crianças ocorra de forma adequada, promovendo assim condições de saúde para um desenvolvimento hígido.

\begin{abstract}
Purpose: to check interfering aspects in the feeding standard of children from 17 to 25 months. Methods: data of speech language pathology evaluation related to 14 children that attended a day-care center in the city of Bauru/SP. A questionnaire was applied in order to get information about general health conditions and neuromotor development, natural or artificial feeding, oral habits and breathing problems. The analysis was made under the following aspects: morpho-functionality of the physicianphysiological characteristic constituting the stomatognathic system and chew and deglutition. The results were submitted to a Descriptive Statistical Analysis. Results: alteration in diverse aspects of the health and the development in children (92.8\%) was ascertained; and also malocclusion (28.6\%), morphological alterations for lips (21.4\%) and tongue (21.4\%); tonicity alterations of cheeks (57.1\%), mento $(21.4 \%)$ and lips (42.8\%); oral or oronasal breathing (42.8\%); dysfunction in chewing (35.7\%) and in swallow solid (50\%) and liquid (42.8) foods. Conclusion: for this group it was observed that the oral habits, morphological and tonicity alterations influence the standard of feeding for the analyzed children, evidencing the need to prevent and detect such factors precociously so that the feeding in the children may occur in an adequate form, thus promoting conditions towards healthful development.
\end{abstract}

KEYWORDS: Feeding Behavior; Child; Child Development; Health Promotion

\section{REFERÊNCIAS}

1. Organização Pan-Americana de Saúde. Organização Mundial da Saúde. Encuesta sobre enseñanza de la lactancia materna en escuelas universitarias de América Latina. Relatório mimeografado da OPS; 1993.
2. Bonati M, Campi R. Breastfeeding and infant illness. Am J Public Health. 2000 Sep; 90(9):1478-9. 3. Andrade CRF. Ações fonoaudiológicas na saúde maternoinfantil. In: Andrade CF, organizador. Fonoaudiologia em berçário normal e de risco. São Paulo: Lovise; 1996. p.25-42.

4. Neiva FCB, Cattoni DM, Ramos JLA, Issler 
H. Desmame precoce: implicações para o desenvolvimento motor-oral. J Pediatr. 2003; 79(1):7-12.

5. Leite ICG, Rodrigues CC, Faria AR, Medeiros GV, Pires LA. Associação entre aleitamento materno e hábitos de sucção não-nutritivos. Rev Ass Paul Cir Dent. 1999; 53(2):151-5.

6. Horwood JL, Fergusson DM. Breastfeeding and later cognitive and academic outcomes. Pediatrics. 1998; 101(1):e9.

7. Silva AM, Silva KP, Oliveira RCPS, Ferreira AJ. A influência da alimentação na mastigação das crianças. Rev Fonoaudiol Bras. 2004; 4(1):1-3.

8. Delgado SE, Halpern R. Possibilidade de intervenção fonoaudiológica na facilitação do vínculo mãe-bebê, através do aleitamento materno. Pediatr Mod. 2003; 39(3):53-7.

9. Miller MJ, Kiatchoosakun P. Relationship between respiratory control and feeding in the developing infant. Semin Neonatol. 2004; 9(3):221-7.

10. Bühler KEB, Limongi SCO. Fatores associados à transição da alimentação via oral em recémnascidos pré-termo. Pró-Fono. 2004; 16(3):301-10.

11. Rocha IE. Avaliação de alguns parâmetros fisiológicos em recém-nascidos prematuros em posicionamento ventral. Cad C Univ. São Camilo. 2003; 9(2):86-95.

12. Soares MEM, Giugliani ERJ, Braun ML, Salgado ACN, Oliveira AP, Aguiar PR. Uso de chupeta e sua relação com o desmame precoce em população de crianças nascidas em Hospital Amigo da Criança. J Pediatr. 2003; 79(4):309-16.

13. Pereira LF, Silva AMT, Cechella C. Ocorrência de hábitos orais viciosos e distúrbios fonoarticulatórios em indivíduos portadores de deglutição atípica. Pró-Fono. 1998; 10(1):56-60.

14. Moss ML, Salentijin L. The primary role of functional matrices in facial growth. Am J Orthod. 1969; 55(6):566-77.

15. Subtelny JD. Effect of diseases of tonsils and adenoids on dentofacial morphology. Ann Otol Rhinol Laryngol. 1975; 84(2Pt2Suppl19):50-4

16. Vieira GO, Silva LR, Vieira TO, Almeida JA, Cabral VA. Hábitos alimentares de crianças menores

RECEBIDO EM: 17/06/2008

ACEITO EM: 20/01/2009

Endereço para correspondência:

Ana Paula Nicolielo

Rua Luiz Aleixo, 5-27

Bauru - SP

CEP: $17013-590$

E-mail: anapaolanicolielo@yahoo.com.br de 1 ano amamentadas e não-amamentadas. J Pediatr. 2004; 80(5):411-6.

17. Altmann EBC, Ramos ALNF, Khoury RBF. Avaliação fonoaudiológica. In: Altmann EBC. Fissuras labiopalatinas. Carapicuíba: Pró-Fono; 1997. p.325-66.

18. Pena CR, Pereira MMB, Bianchini EMG. Características do tipo de alimentação e da fala de crianças com e sem apinhamento dentário. Rev CEFAC. 2008; 10(1):58-67. dx.doi.org/ S1516-18462008000100009.

19. Marchesan IQ. Correção forma-função: sistema estomatognático. In: Marchesan IQ. Motricidade oral: uma visão clínica do trabalho com outras especialidades. São Paulo: Pancast; 1993.p. 25-34. 20. Fundo das Nações Unidas para a Infância (UNICEF). [homepage na internet] Brasília. [acesso em 20 set 2006] Disponível em: URL: http://www. unicef.org/br

21. Companhia de Internet Bibliomed. Informações sobre saúde. Pesquisa de medicamentos genéricos. [homepage na internet] Belo Horizonte: Bibliomed Inc; 1998. [acesso em 15 out 2006] Disponível em: URL: http://boasaude.uol.com.br/GENERICOS/ showdocs.cfm? $\mathrm{id}=28$

22. American Academy of Sleep Medicine. Diagnostic classification steering commitee. [homepage na internet] American Academy of Sleep Medicine; 2008. [acesso em 18 set 2008] Disponível em: URL: http://www.aasmnet.org/

23. Lourenço EA, Lopes KC, Pontes Júnior A, Oliveira MH, Umemura A, Vargas AL. Estudo comparativo radiológico e nasofibroscópico do volume adenoideano em crianças respiradoras orais. Rev Bras Otorrinolaringol. 2005; 71(1):23-8

24. Peres KGI, Barros AJD, Peres MA, Victora CG. Efeitos da amamentação e dos hábitos de sucção sobre as oclusopatias num estudo de coorte. Rev Saúde Pública. 2007 Jun; 41(3):343-50.

25. Ignacchiti PR, Gesualdi KC, Cursage FPC, Almada RO. Hábito de sucção de chupeta e mordida aberta anterior na criança com dentição decídua. Rev CEFAC. 2003; 5(3):241-5.

26. Siqueira ABUM. A época de instalação da mamadeira esta relacionada com a instalação de hábitos orais não-nutritivos? Rev CEFAC. 2003; 5(4):313-6.

27. Guedes ZCF. Algumas considerações fonoaudiológicas sobre deglutição atípica numa abordagem multidisciplinar. Acta Awho. 1985; 2(4):40-6.

28. Marchesan I. Uma visão compreensiva das práticas fonoaudiológicas: a influência da alimentação no crescimento e desenvolvimento crânio-facial e nas alterações miofuncionais. São Paulo: Pancast; 1998. 238p. 